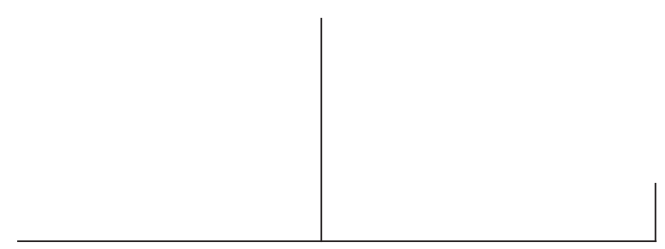

Rev. Latinoam. Psicopat. Fund., III, 1, 131-142

\title{
La paradoja y lo negativo Aportes epistemológicos para la Psicopatología Fundamental*
}

\author{
Flora Singer
}

Se trata de una investigación sobre epistemología psicoanalítica que tiene como marco de referencia teórico el pensamiento francés contemporáneo. Su tesis central es la alteridad ontológica del inconsciente, que se extiende a la teoría mediante una lógica que denominamos paradojal y de lo negativo.

Esta lógica paradojal permite aprehender los contrarios constituyentes de la alteridad como complementarios y no excluyentes: conservan su especificidad pero hay también pasaje y transformación de uno en otro.

Este pasaje en la transformación plantea un límite al saber: éste no puede ser total. En ese contexto, definimos lo negativo como lo que hace límite al saber, ya sea porque a nivel del inconsciente nos topamos con el enigma, el ombligo, o a nivel de la teoría, porque una noción, un sentido, devienen otro.

Consideramos que este abordaje integrativo de la alteridad puede proveer elementos de análisis útiles para el relacionamiento de la teoría con la clínica, el relacionamiento interteórico, el interdisciplinario, y finalmente para la propia metodología de la investigación.

Palabras llave: Epistemología psicoanalítica, paradoja, negativo,

psicopatología fundamental

* Trabajo acerca de la orientación de mi investigación, expuesto en la IV Reunión Científica de la Red Universitaria de Investigación en Psicopatología Fundamental, São Paulo, octubre 1998. 


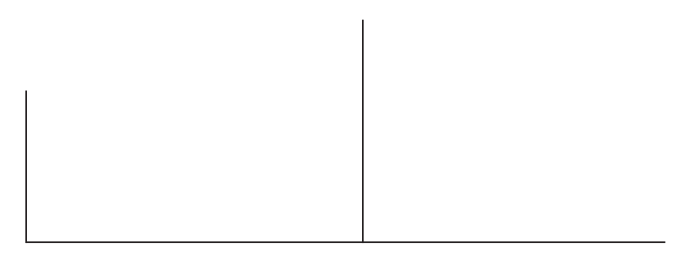

El inconsciente es lo otro que la conciencia. Este postulado, simple en apariencia, determina el estatuto del saber en psicoanálisis, y sus derivaciones sientan bases para una epistemología psicoanalítica.

Históricamente la reflexión epistemológica en psicoanálisis ha sido tributaria de criterios extrínsecos al mismo, que corresponden a una tradición positivista y no se adaptan a su naturaleza. Lo que es común en ellos es la búsqueda de una mismidad que permite el ordenamiento y axiomatización del conocimiento, mediante el cogito como ideal de sujeto idéntico a sí mismo, y el principio de identidad y de no contradicción.

El pensamiento francés contemporáneo en psicoanálisis y en filosofía provee de instrumentos teóricos sumamente fértiles para la reflexión en torno a una epistemología psicoanalítica.

Dicho pensamiento en psicoanálisis hace énfasis en el inconsciente como radical alteridad, y da lugar al ombligo, al punto de lo desconocido, también como teniendo un lugar específico. Este paradigma psicoanalítico se inserta en un paradigma más vasto, que desde la filosofía y las ciencias humanas cuestiona los criterios de cientificidad positivistas, $\mathrm{y}$ provee una base teórica en torno a la alteridad.

La llamada corriente de la discontinuidad, integrada por G. Bachelard, G. Canguilhem y M. Foucault, se muestra extremadamente fértil en ese sentido. Su crítica al positivismo 


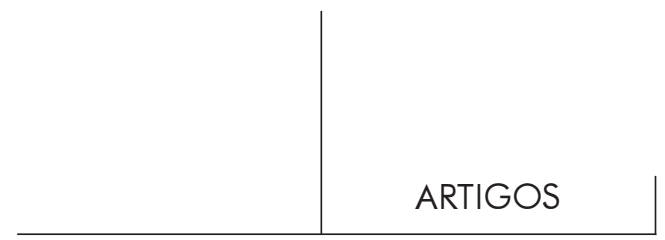

permite hacer emerger el campo de las ciencias humanas en su especificidad: campo de lo paradojal, lo singular, lo heteróclito, lo indeterminado. Hay también en estos pensadores una crítica al concepto y mediante ella, una necesidad de hacer estallar el ser, lo inmutable, y lo idéntico. La lógica del significante de J. Lacan va en ciertos aspectos, en el mismo sentido. Junto con la influencia de la lingüística y el estructuralismo, ello contribuye a que el psicoanálisis se desplace de una zona de influencia marcada por criterios cientificistas y positivistas a otra, derivada de las ciencias humanas, que busca abordar algo que permanece como resto en un cierto ideal de racionalidad. No obstante, no es de irracionalidad de lo que se trata, sino de otra racionalidad.

Cuando decimos que el inconsciente se constituye como alteridad en relación a la conciencia, hablamos de un aspecto ontológico, en relación a la materia con la que trabajamos en la clínica, de un aspecto teórico o de cómo poner a trabajar las nociones, y de una serie de consecuencias en relación al saber sobre dicho inconsciente. Estos aspectos son isomórficos entre sí.

La teorización en psicoanálisis no alcanza totalmente su objeto, y guarda un límite en relación al saber ligado al punto en el que el inconsciente se constituye como irreductible a la consciencia. El saber guarda siempre un reducto de no saber.

Por otra parte, las nociones psicoanalíticas, para acompañar a un objeto que escapa, que las excede, poseen una transformabilidad, un devenir otras, por la cual no se dejan aprehender en definiciones precisas. Esa alteridad que las constituye guarda una correspondencia con la alteridad del inconsciente. Primera y segunda tópica, sadismo-masoquismo, o aún, la evolución del concepto de yo son otros tantos ejemplos donde esas nociones se empobrecen fuera de una perspectiva dinámica que debe tener en cuenta la transformación de la noción en el interior de la teoría, como si la estaticidad de un concepto no alcanzara para delimitar su objeto.

La estructura lógica de la paradoja - que es la del inconsciente y la de su teoría - permite la inclusión y complementariedad de elementos diferentes y aún opuestos, que coexisten en su diferencia y se interrelacionan sin perder cada uno su especificidad. Llamamos paradojal en el caso de las nociones psicoanalíticas, toda dinámica en la cual hay transformación de la significación de un término en otra, pero no obstante siguen coexistiendo todas las significaciones, diferentes, y aún, opuestas.

En psicoanálisis necesitamos incluir la diferencia en la medida en que el inconsciente es la diferencia (o la alteridad). La incluimos mediante lo que denominamos una lógica de la paradoja y lo negativo.

El saber paradojal es un saber que no termina de saberse. Metodológicamente ese no-saber debe ser integrado al sistema, no como lo hace el positivismo, bajo la forma de aspiración a su eliminación, sino por el contrario, de poder hacerlo circular, y poder aprehenderlo en la pluralidad dinámica de sus formas posibles, incluida 


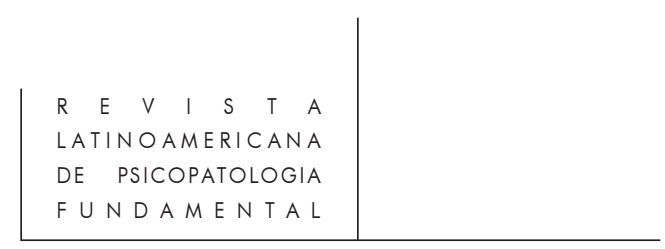

también la de un no-saber irreductible. En ese sentido, la integración del no-saber concierne menos la posibilidad de su inversión que el juego dinámico de fuerzas, juego paradojal, del saber y el no-saber en el interior de una estructura o elemento del dispositivo psicoanalítico, clínico o teórico. Llamamos negativo a ese límite al saber, en dos aspectos: en relación a la cosa, y en relación a la teoría.

1) En relación a la cosa constituye el límite ligado al punto de detención del saber frente al ombligo, al enigma, a lo real en busca de sentido.

2) En relación a la teoría constituye el límite ligado al punto de transformación del sentido, en donde una noción deviene otra, y ese devenir es paradojal en la medida en que ese punto de transformación escapa en último término a su captación por el logos.

Que se trate de reconstruir el movimiento que condujo a Freud de la primera a la segunda teoría de la angustia, no quiere decir que se puedan restablecer los eslabones que hacen que la primera teoría de la angustia anticipe una segunda, y que pueda determinarse en qué punto la segunda ya-esté-ahí a partir de un cierto momento de la primera. Esta indeterminación en términos de saber forma parte de la naturaleza de la paradoja. El otro ya está ahí en lo mismo, y esta mezcla funda doblemente lo mismo y lo otro. Se puede pensar en esos términos la intrincación compleja del amor y el odio en las diferentes figuras psicopatológicas, en donde el amor no lo es sin odio y viceversa. En la clínica, el estatuto de la interpretación es menos el de una hermenéutica, que el de una dirección en el abanico de los posibles.

Tanto lo real como imposible, como el universo del sentido como posible se constituyen como indecidibles en relación al saber.

La cualidad del discurso depende del tipo de objeto al que se refiere. Esto no es accesorio. Por el contrario, que la cosa y el lenguaje con el que se designa tengan o no las mismas propiedades tiene consecuencias prácticas. Es el propio dispositivo para pensar en psicoanálisis que es así interpelado, y con él, la manera de producir teoría, de hacer clínica, y finalmente, es interpelada también la manera de relacionarse el psicoanálisis con otras disciplinas.

Mi experiencia como profesora de psicoanálisis en la universidad se vio atravesada constantemente por la interrogación - mía, de mis estudiantes - acerca de la relación de la teoría con la clínica, y la relación entre teorías. El psicoanálisis no puede prescindir de una lógica estática que eleva conceptos relativamente definidos a un estatuto axiomático, y que permite delimitar un campo teórico y conformar la coherencia interna de la teoría. El Edipo, los fantasmas originarios, o la lógica del significante en la teoría lacaniana, así como otras nociones en las teorías freudiana, kleiniana u otras, permiten recortar de una cierta manera los fenómenos clínicos. Estas nociones de carácter axiomático tornan al inconsciente inteligible, pero arriesgan también, ya sea capturar la singularidad de la clínica en las redes de la teoría, o dogmatizar estas nociones despojándolas de su movimiento metafórico. El exceso 


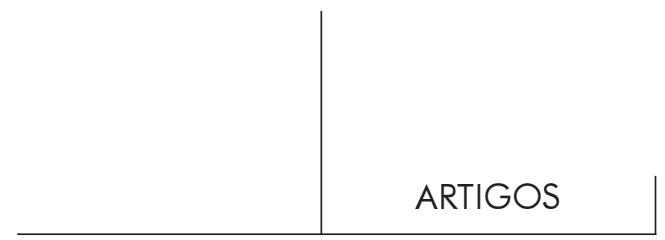

de teorización amenaza siempre la necesaria ambigüedad del psicoanálisis, atrapándolo en sistemas teóricos cerrados.

Estas nociones que poseen el estatuto de universales son necesarias, pero guardan una relación dilemática con la clínica y con el movimiento necesario a la teoría, a menos que medie un espacio de diferenciación de los universales en relación a la singularidad de la situación clínica, y un espacio metafórico por el cual estas nociones puedan ser dinamizadas y paradojalizadas. De otra manera, la utilización de una lógica estática puede contribuir, por la fijación de las nociones, a un terrorismo teórico, incluso en la propia clínica.

Por otro lado el movimiento metafórico propio a la paradoja permite la dinámica constructivo/deconstructiva propia a la producción del saber por la cual cada teoría puede ser prolongada y resignificada. Permite también el trabajo metapsicológico propio a la clínica. Promueve la dinámica inter-teórica y la alternancia y complementariedad en los lugares del saber. De esta manera, la verdad no es propia a una teoría. Pero así como una lógica estática genera como efecto de sentido la dogmatización de las nociones, el movimiento paradojal de las mismas tiene también efectos de sentido, los propios a la diseminación, tomando el término de J. Derrida: sentidos nuevos, no del todo determinables, que contribuyen a la creación teórica pero también al "ruido", a la dispersión, a la desviación del tronco teórico fundador.

Creemos importante distinguir entonces entre la teoría y las lógicas en las cuales ésta se vehiculiza. Estas lógicas, estática una, dinámica otra, transitan en el lenguaje y causan efectos de sentido no del todo determinables.

En relación a la clínica, la "cosa" toma la delantera y habilita a la teoría a enunciarla de varias maneras. No obstante esas varias maneras no son cualquier manera, y una lógica de lo paradojal y lo negativo, que subtiende a la clínica, debe ser explicitada una y otra vez. Pues el riesgo es constante, de que el lenguaje tome la delantera en relación a la cosa, la reifique, la atrape en las redes del logos.

El concepto como instrumento lógico alcanza en su designación a la cosa: la define, la circunscribe como totalidad. En esa medida conserva un peso ontológico. Su estaticidad y la claridad de su definición también contribuyen a ello.

En psicoanálisis, la movilidad de la cosa hace que pueda ser dicha de más de una manera. Esa movilidad haría que habláramos más de metáfora que de concepto. Pero hay además una cualidad particular en esa metáfora. No sólo es transporte, movilidad paradojal, sino que es transporte de lo negativo.

Tradicionalmente la metáfora como figura lingüística, en su movilidad, pone de manifiesto homologías, equivalencias, y más los momentos de parada que los de tránsito. Los desarrollos de P. Fédida rescatan de la metáfora el transporte, el devenir otro, en relación al cual el contenido representativo es un momento segundo. Jerarquizan así el movimiento de la metáfora sobre su estado final, y más lo que ésta acarrea de nuevo que lo que instaura en la serie de las equivalencias. Una vez 


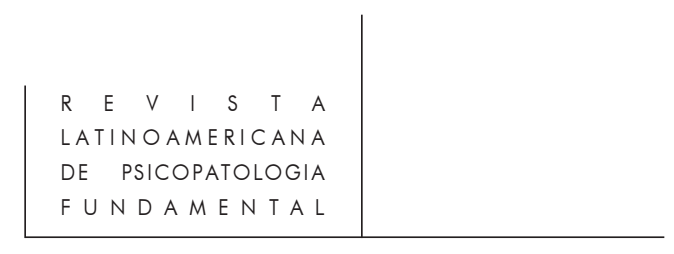

más, esta vez en relación a la metáfora, se prioriza la alteridad, la alteridad como dinámica y como corte. Todo pasaje entre lo mismo y lo otro integra también un corte virtual, una différance. En el espacio de transformaciones metafóricas se introduce el circuito paradojal que pone en evidencia, más que lo que hay de lo mismo en la metáfora, lo que hay de diferente; más el engendramiento de lo nuevo que la reduplicación de la representación.

Que el inconsciente es un otro debe ser preservado en el plano del lenguaje. La designación de ese otro no sólo es una metáfora, sino una metáfora que en lugar de acarrear una homología, acarrea algo de una alteridad nunca del todo alcanzada. En esa medida, la metáfora es transporte de lo negativo. Transporte de lo otro a partir de lo mismo, se ubica así en el entre-dos de la paradoja. Y eso, doblemente: producción de un sentido primero a partir de lo real y la falta de sentido, o producción de un sentido nuevo a partir de un sentido anterior.

En el primer caso, que pueda establecerse un espacio de diferenciación, metafórico, entre la cosa y su designación primera, por ejemplo un material que emerge en la sesión y su integración en un universo de sentido, permite que la cosa continúe preservando su negatividad y su coseidad más allá de su designación, y que esa designación conserve su estatuto de ficción. Y ello tiene consecuencias clínicas. No se trata de una nueva clínica, sino de la explicitación de los presupuestos lógicos de esa teoría en acto en la clínica, teoría que positiviza los fenómenos quitándoles su paradojalidad, o por el contrario, acompaña el movimiento de la paradoja y lo negativo.

En nuestro artículo "El duelo, ¿qué modelización?" desarrollamos cómo puede haber en la situación psicopatológica del duelo subrepticiamente una positivización o negativización de la teoría subyacente que incide en su abordaje terapéutico. Hay una lectura del texto freudiano, en donde las nociones de "trabajo de duelo", "elaboración", "objeto sustitutivo", jerarquizan más la positividad del estado final y el duelo ya elaborado, que el proceso mismo, productivo, psicopatológico, del duelo. En ese abordaje asimismo, la relación de objeto con el muerto arriesga caer en una economía sustitutiva por la cual hay desinvestidura del muerto y sustitución por otro objeto. Pero en realidad lo que en el duelo se impone al psiquismo, como dato primero, no es una ausencia sino una realidad otra. Y el muerto no es desinvestido, sino que es reinvestido en un lugar otro. Lo negativo está connotado desde los dos sentidos que le hemos atribuido: la muerte como realidad otra, anterior a toda elaboración, aún en búsqueda de sentidos y de representaciones, y está constituido también por los numerosos espacios-entre involucrados y por su dinámica paradojal (objeto externo-interno, libido de objeto-libido narcisista, pulsión de vida-pulsión de muerte). Ello da la posibilidad de ficcionar ese lugar virtual de lo otro, (no lo ausente) que es investidura de la muerte, y abrir una perspectiva clínica distinta. Se articula además así duelo y melancolía, normal y patológico, en su complementariedad paradojal. 


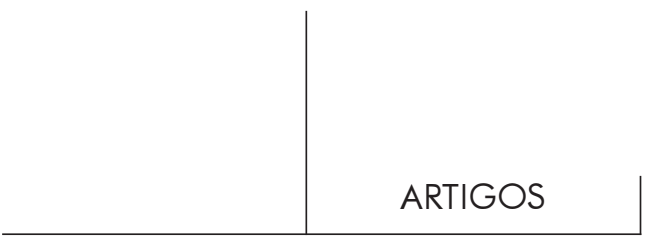

Que lo negativo no es una ausencia, significa que tiene otra forma de presencia y que debe poder ser acogido como tal. Plantea que la propia materia del inconsciente es la de la paradoja; que nuestra escucha del masoquismo, por ejemplo, debe también permitir la escucha del masoquismo como otro sadismo, o la presencia de lo sádico en otro lugar.

Si en cada psicoanálisis hay una teoría individual en acto, la del paciente con sus síntomas y la del analista, la trama de esa teoría, y la manera de entretejerla, tienen que ver con una forma de disponer del dispositivo a pensar y con ciertas lógicas que ponen a trabajar la teoría de una cierta manera. Los deslizamientos lógicos en la clínica pueden producir una reificación de las nociones, o una confusión de los niveles normativo y de existencia, u otra serie de disfuncionamientos de la teoría en relación al material. Nuestras intervenciones clínicas no sólo están comprometidas con referentes teóricos - kleiniano, lacaniano etc. - sino también impregnadas de presupuestos lógico-metodológicos que causan parasitajes, y pueden y deben ser desmontados y explicitados. Por eso creemos que nuestra investigación no sólo tiene implicaciones teóricas sino también clínicas.

Volviendo al plano teórico, cuando la metáfora es transporte entre dos sentidos, y además de las homologías acarrea también algo de lo otro, no hay reversibilidad posible entre los sentidos. El pasaje de la noción de significante del universo lingüístico al psicoanálisis constituye un ejemplo de ello. En ese pasaje hay una heterogeneidad que se instaura, por la que no se trata del mismo significante. Cuando éste connota aspectos específicos al campo psicoanalítico, ya la serie de las différances lo aleja del campo lingüístico.

Los matemas: ¿son fórmulas matemáticas o metáforas? Discusión falaciosa, ni lo uno ni lo otro. Por obra de la metáfora, el matema, entendido desde su valencia interna al campo psicoanalítico, se torna una ficción, con un potencial evocador de lo negativo de la cosa.

Es importante distinguir nociones tomadas de otras disciplinas, de la lógica en la cual son vehiculizadas. El pasaje al campo psicoanalítico requiere que esas nociones sean paradojalizadas y negativizadas, y adquieran así una valencia específica a dicho campo. El riesgo de estos pasajes es que sean hechos desde una lógica de lo mismo y no de lo otro.

El referente de toda noción en psicoanálisis es un referente negativo. No guarda una relación directa con su nominación. Entre uno y otro, un espacio de heterogeneidad debe preservarse para que una reificación de la cosa no tenga lugar.

Las nociones como ficciones permiten mantener el estatuto otro de la cosa. La pulsión es un ejemplo, que ofrece el interés de abrirse a la cuestión del relacionamiento inter-disciplinario.

Cuando se define la pulsión como límite entre lo psíquico y lo somático, hay una posición en el debate científico que intenta favorecer con esa definición la relación 


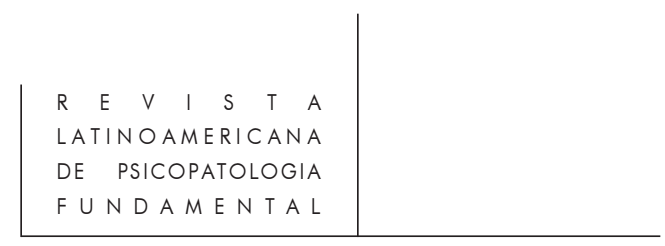

entre psicoanálisis y biología, tomando la pulsión como llave de paso común a ambas disciplinas.

Varios deslizamientos positivizantes subyacen a esa argumentación:

En primer lugar la pulsión es tomada como cosa, como reificación de una noción que es una ficción. La pulsión no es un ente de existencia, es una noción con un poder ficcional que señala un ente en negativo. Esa ficción es interna al campo psicoanalítico y adquiere significación en un haz de relaciones y en una estructura fuera de los cuales no tiene la misma significación.

En segundo lugar, las llaves de paso o elementos comunes entre el psicoanálisis y otras disciplinas se apoyan en una lógica de la mismidad, y su pertinencia metodológica debe ser explicitada y evaluada. Una lógica de la paradoja permite como alternativa, esclarecer y ver la pertinencia de buscar elementos comunes, o por el contrario, de establecer su red diferencial. Al lado de metodologías que buscan analogías o elementos comunes se hace necesario también una metodología opuesta, de búsqueda de diferencias.

En tercer lugar, con esta búsqueda de diferencias, la noción misma de límite es puesta en cuestión. Entre la conciencia y el inconsciente la frontera no es precisa. Está constituída por una zona no determinable de pasaje y transformaciones entre lo uno y lo otro. Lo mismo ocurre con las nociones, y de la misma manera con las fronteras del psicoanálisis. Para que un pasaje fuera posible del campo psicoanalítico al biológico, esa frontera debería ser concebida como una línea clara, de pasaje en la contigüidad. Desde la paradojalidad constituyente del psicoanálisis, sus límites son menos zonas definidas que una zona-entre de heterogeneidades que hacen a intercambios no del todo determinables.

Entre lo uno y lo otro hay espaciamiento, y ese espaciamiento constituye también otro tipo de límite. La pulsión opera como concepto límite al psicoanálisis. No quiere decir que sea un límite que comunique $a$ otra disciplina, ni tampoco que sea un concepto límite de esa otra disciplina. Es un límite interno al campo psicoanalítico.

Estas modelizaciones no dichas, deben ser explicitadas en base a elementos epistemológicos específicos al psicoanálisis.

El psicoanálisis convoca la cuestión del otro. Ello determina una epistemología de la alteridad, y es en ese contexto que se inserta también la cuestión de la relación al otro inter-teórico, y aún, al otro interdisciplinario.

En el dispositivo analítico, un espacio vacío debe poder acoger la subjetividad del otro. Dice P. Fédida en "Crise et contretransfert" (p. 31): "Como el dispositivo psicoanalítico lo muestra de manera paradigmática, el encuentro con el otro, que es la radical alteridad que marca el encuentro con toda subjetividad, debe ser acogido en un espacio en donde tenga cabida esa distinción de escenas, y donde el encuentro con la palabra pueda también abrirse al vacío de la no palabra". Creemos que este 


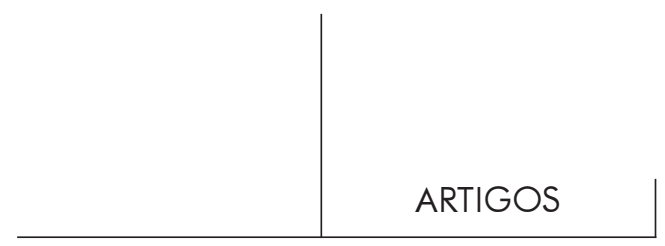

dispositivo se refiere también a la Psicopatología Fundamental. Chora es ese lugar vacío, sitio del lenguaje, que permite el transporte metafórico y habilita un encuentro con lo otro.

La Psicopatología Fundamental constituye ese espacio vacío - tal chora - que permite la emergencia y el relacionamiento con lo otro a nivel inter-teórico e interdisciplinario, en torno a un tema, el pathos, el sufrimiento humano.

Lo vacío no es la ausencia. Así como a partir del lugar vacío del dispositivo analítico hay producción de un nuevo saber (de sí), de la misma manera, en el espacio vacío de la Psicopatología Fundamental, lo mismo y lo otro pueden relacionarse, y algo nuevo como saber puede emerger. Por medio de un acto de nominación Psicopatología Fundamental - un lugar de emergencia de teorías y textos es así creado. Cuál es la ley de su regulación, y los principios que fundan esa emergencia, son consecuentes con una lógica de la paradoja y de lo negativo.

En efecto, en ese lugar vacío, los distintos discursos en torno al pathos se ponen en relación. El tipo de saber que de allí emerge no es un saber ecléctico. Hay articulación y no superposición de saberes. Esa articulación no toma la forma de la unificación sino de intercambios con zonas de intersección, de cruzamiento y de mantenimiento de las heterogeneidades relativas.

En ese intercambio que la Psicopatología Fundamental promueve, además de relaciones en torno a elementos comunes, se confrontan diferencias relativas, y esa coexistencia de puntos de vista es también aceptación de la paradoja. La aceptación de la paradoja lo es doblemente: por la coexistencia de saberes diferentes, y por el principio que subyace a la Psicopatología Fundamental, que articula lo normal y lo patológico como siendo opuestos complementarios, en solución de continuidad y no de exclusión.

En ese intercambio, hay un espacio para saberes relativos así como para el enigma, y para modelizaciones específicas y singulares, así como es singular en última instancia el saber sobre el pathos de cada sujeto.

Se produce así un saber nuevo, y también un saber sobre las fronteras y los límites de cada campo. Los resultados del diálogo inter-teórico e interdisciplinario no pasan necesariamente por una incorporación de saber en cada campo, sino también por una ampliación y afinamiento de las zonas-entre en donde tienen lugar las différances, lo que indirectamente alimenta el saber de cada campo.

El aporte de la modelización del psicoanálisis a este tipo de producción del saber, lo es a partir de la cualidad de su objeto, de su referencial paradojal, y de la integración de lo negativo. El psicoanálisis aporta la visualización de las zonas-entre, las que superan los límites que cada disciplina se otorga a sí misma. Por las características de su objeto y de su reflexión, el psicoanálisis aporta una experiencia de extra-territorialidad. Lo inter-teórico, lo inter-disciplinario, deben también ser comprendidos como "extra" teórico o "extra" disciplinario: como saber posible que 


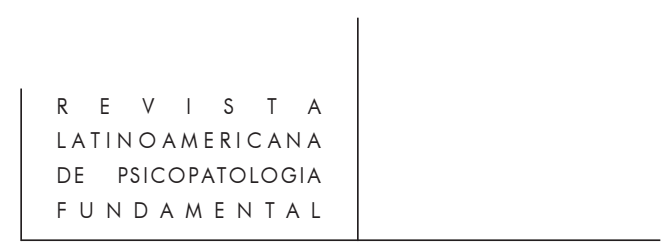

va más allá de los saberes particulares. Decíamos en nuestro artículo "Modelos en psicoanálisis", que "La epistemología que se desprende del psicoanálisis compromete una relación con la alteridad, no sólo en el seno del psicoanálisis, sino en relación a los objetos de conocimiento, a los modelos, y como relacionamiento inter-científico. Este modo de articulación integra el saber posible, pero también el saber imposible. Es fractura del saber, pero también... constitución de un saber nuevo", que compromete asimismo el límite al saber.

Nociones tales como metáfora o chora, tal como utilizadas por P. Fédida, tienen un estatuto particular dentro del discurso. No transportan significación, no se dicen a ellas mismas. Señalan un lugar vacío, como chora, o permiten que la dinámica paradojal se ponga en marcha, como la metáfora. No pertenecen al campo de significancia psicoanalítica. Transcurren en un plano metateórico, externo a la teoría. Ello permite hacer alusión a lo negativo y a la paradoja preservándolos al mismo tiempo como un territorio otro. Como el lugar vacío del damero, permiten circunscribir el territorio de lo negativo en el cual la paradojalidad de las otras nociones puede desplegarse. Permiten así que se implante una cierta modelización y una cierta lógica.

Denominamos estas nociones operadores. No hacen alusión a entes sino a intersticios, a zonas-entre, así como a la dinámica que los pone en relación. Su nominación no es exhaustiva, y distintos elementos pueden tomar la función de la metáfora o de chora.

La Psicopatología Fundamental tiene un estatuto semejante al de chora: hay un acto de nominación que crea un lugar - no una cosa, ni una nueva disciplina. La cosa, o la disciplina, es positividad, pleno. Con el lugar, lo negativo es preservado como espacio para acoger lo otro. Ese lugar provee un dispositivo para pensar la psicopatología que permite un movimiento y una producción en el orden de la teoría. Por eso ese dispositivo está constituido por una lógica paradojal y de lo negativo.

Una reflexión epistemológica sobre el psicoanálisis puede contribuir a poner en relación y clarificar sus diferentes niveles teóricos y prácticos, o sea, discursivos, y los niveles lógicos y metodológicos que atraviesan las teorías. Puede contribuir a regular los intercambios entre teoría y clínica, y entre las diferentes teorías, en el interior del campo psicoanalítico y en relación a otras disciplinas.

Esta reflexión sólo puede desarrollarse a partir del reconocimiento de una lógica paradojal y de lo negativo como instrumento de pensamiento adecuado a la dinámica del inconsciente.

Intentamos que esta reflexión no permanezca en un nivel exclusivamente teórico sino que se pueda promover su aplicación, fundamentando una metodología de trabajo y herramientas lógicas que contribuyan a la reflexión en psicoanálisis.

Cuando a su vez el psicoanálisis se integra al ámbito universitario y específicamente a la investigación, esta explicitación de sus presupuestos y 


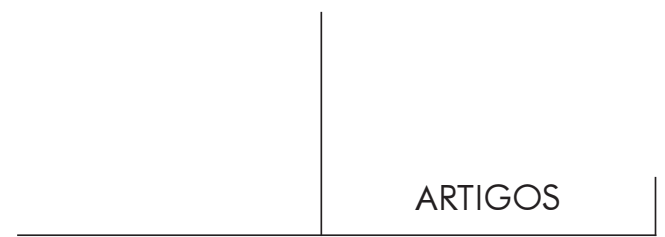

especificidad puede contribuir al afinamiento de la metodología de la propia investigación, realimentando ésta con nuevos elementos.

Bibliografia

DerridA, J. La dissémination. Paris: Seuil, 1972.

FÉDIDA, P. “Théorie des lieux”. Psychanalyse à l’Université. № 53 y 56, Paris, 1989.

"Tradition tragique du psychopathologique. A propos du pathei mathos de

l'Agamemnon", in Crise et contre-transfert. Paris: PUF, 1992.

"D'une psychopathologie générale à une psychopathologie fondamentale. Note sur la notion de paradigme", in Crise et contre-transfert, op. cit.

"Théorie des lieux dans la psychanalyse. Sur la transformabilité métapsychologique du modèle". Colloque de la Revue Internationale de Psychopathologie. Paris: PUF, 1995.

Singer, F. "Modelos en psicoanálisis". Revista Latinoamericana de Psicopatologia Fundamental, vol. I, nº 2, São Paulo, 1998.

"El duelo: ¿qué modelización?". Revista Latinoamericana de Psicopatologia Fundamental, vol. II, no 1, São Paulo, 1999.

"Psychopathologie et paradoxalité épistémologique de la psychanalyse", tesis de

Doctorado. Presses Universitaires du Septentrion, Lille, 1999.

Resumos

Trata-se de uma investigação sobre epistemologia psicanalítica, tendo como marco de referência teórica o pensamento contemporâneo francês.

A tese principal é a alteridade ontológica do inconsciente, que se estende para a teoria por meio de uma lógica que denominamos paradoxal e do negativo. Esta lógica paradoxal permite apreender os opostos constituintes da alteridade, como complementários e não excludentes: conservam sua especificidade, porém também têm passagem e transformação de um em outro.

Esta passagem na transformação coloca um limite ao saber: este não pode ser total. Nesse contexto, definimos o negativo como aquilo que faz limite ao saber, seja porque ao nível do inconsciente deparamo-nos com o enigma, o umbigo ou, ao nível da teoria, porque uma noção, um sentido, torna-se outro.

Consideramos que esta abordagem integrativa da alteridade pode dispor de elementos de análise úteis para o relacionamento da teoria com a clínica, o relacionamento interteórico, o interdisciplinar e, finalmente, para a própria metodologia da investigação.

Palavras-chave: Epistemologia psicanalítica, paradoxo, negativo, psicopatologia fundamental 


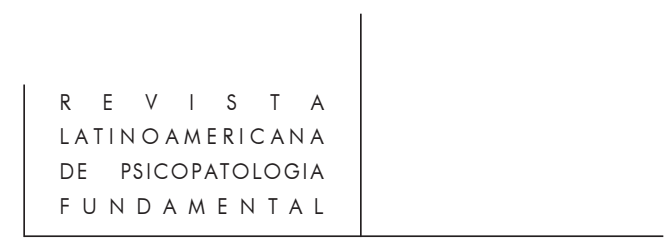

Il s'agit d'une recherche d'épistémologie psychanalytique, qui a comme cadre de référence théorique la pensée francaise contemporaine.

Sa thèse centrale est l'altérité ontologique de l'inconscient, qui s'étend à la théorie par le biais d'une logique que nous nommons paradoxale et du négatif.

Cette logique paradoxale permet de saisir les contraires constitutifs de l'altérité, comme étant complémentaires et ne s'excluant pas: ils conservent leur spécificité mais il y a aussi passage et transformation de l'un dans l'autre.

Ce passage dans la transformation pose une limite au savoir: celui-ci ne peut pas être total. Dans ce contexte, nous définissons le négatif comme étant une limite au savoir, soit parce qu'au niveau de l'inconscient nous nous trouvons face à l'énigme, à l'ombilic, soit parce qu'au niveau de la théorie, une notion, un sens, deviennent autre.

Nous considérons que cet abord intégratif de l'altérité peut pourvoir des éléments d'analyse utiles pour la mise en rapport de la théorie avec la clinique, les rapports inter-théoriques, inter-disciplinaires, et finalement, pour la méthodologie de la recherche elle-même.

Mots clés: Épistémologie psychanalytique, paradox, négatif, Psychopatologie

foundamentaux

This is a research that concerns psychoanalytic epistemology, and its theoretic reference is contemporary French thought.

Its main thesis is the ontologic alterity of unconscious, which is transferred into theory by means of a paradoxality and negativity logic.

This paradoxality logic enables to apprehend the contraries which integrate alterity, as complementary and not excluding: they maintain their specificity but there are passages and transformations between one and another.

This passage which transforms one into another determines a limited knowledge, not a total one. We call negative this limit to knowledge, because of nature of the unknown unconscious, and also because of transformations of senses and notions inside the theory.

This integrative analysis of alterity can provide useful methodologic elements for relating theory and clinics, for inter-theoretic and inter-disciplinary relationships, and also for research.

Key words: Psychoanalytic epistemology, paradox, negative, Fundamental psychopathology 